\title{
What are the long-term effects of smoked marijuana on lung health?
}

\author{
Wan C. Tan MD, Don D. Sin MD
}

Cite as: CMAJ 2018 October 22;190:E1243-4. doi: 10.1503/cmaj.181307

W ith the passage of Bill C-45 (also known as the Cannabis $A c t)$, Canada becomes the second country in the world, after Uruguay, to legalize the use of marijuana for medical and recreational purposes. A commentary published in Science referred to the legislation as a "great ganja experiment." 1 The act aims to "keep cannabis out of the hands of youth; keep profits out of the hands of criminals; [and] protect public health and safety by allowing adults access to safe, legal cannabis." Whereas many view marijuana as a relatively safe drug, and the numerous health benefits and safety of marijuana, especially when used for medicinal purposes, are touted in various media, others have expressed concern that the overall health benefits of "medical" marijuana have been overstated and the drug's harms understated, as was the case for tobacco cigarettes before 1964. ${ }^{3}$ Established health risks of marijuana smoking include dependence syndrome, motor vehicle crashes, effects on adolescent psychosocial development and mental health issues. ${ }^{4}$ We discuss what is known about the effects of smoked marijuana on the lungs and highlight important uncertainties that will need to be addressed through rigorous research.

Before the release of the US Surgeon General's report on smoking and health in 1964, ${ }^{3}$ tobacco advocates glamorized the health benefits of cigarettes by promoting the notion that smoking reduced stress and anxiety, controlled appetite, enabled weight control, enhanced manual dexterity and mental concentration, improved symptoms of ulcerative colitis and osteoarthritis, and prevented Parkinson disease. ${ }^{5}$ We now know that smoking is deadly, and it is widely accepted that the harmful health effects of cigarette smoke are not caused by nicotine.$^{6}$ Cigarette smoke generates more than 4000 other chemicals, including 43 known carcinogens and 400 other toxins, that directly or indirectly induce harm in the airways. ${ }^{6}$ Marijuana is qualitatively similar to tobacco with the exception of the active agent, tetrahydrocannabinol (THC). Chemical analysis reported higher quantities of ammonia, hydrogen cyanide, nitric oxide and nitrogen oxides in marijuana smoke than tobacco smoke. ${ }^{7}$ In vitro studies have shown that marijuana smoke elicits greater oxidative stress, apoptosis and inflammatory responses in lung cells than tobacco. ${ }^{8}$ Intuitively, one would expect that the adverse effects of smoked marijuana

\section{KEY POINTS}

- Some view marijuana as a relatively safe drug with substantial health benefits, whereas others suggest that the long-term harm will outweigh any potential benefits.

- Marijuana is qualitatively similar to tobacco with the exception of the active ingredient, tetrahydrocannabinol.

- Owing to substantial methodological issues, previous epidemiologic and clinical studies examining marijuana smoking on the risk of tobacco-sensitive lung diseases (e.g., lung cancer and chronic obstructive pulmonary disease [COPD]) have not produced consistent results.

- There is a pressing need to understand the long-term effect of recreational and medical marijuana smoking on lung-function decline and risk of lung cancer and COPD.

would at the very least be comparable to that of tobacco smoking. Meta-analyses and systematic reviews ${ }^{9,10}$ generally agree that marijuana smoking causes respiratory symptoms and increases the risk of chronic bronchitis among long-term cannabis smokers. Yet epidemiologic and clinical studies examining marijuana's effect on lung function have yielded mixed results.

Some cross-sectional studies showed that marijuana smokers had a lower forced expiratory volume in one second $\left(\mathrm{FEV}_{1}\right) /$ forced vital capacity (FVC), a measure of airflow obstruction, and impaired large airway function compared with nonsmokers of marijuana. ${ }^{9,10}$ However, three other studies failed to find such an association, instead reporting a paradoxical increase in FVC.,10 To date, five longitudinal studies have also shown conflicting results: no decline in $\mathrm{FEV}_{1}$ in a convenience sample of heavy smokers; a suggestion of gas trapping in a population cohort; and a possible dose-dependent pattern of marijuana exposure and lung-function change. ${ }^{9,10} \mathrm{~A}$ study of four consecutive surveys of nontobacco smokers assumed to be marijuana smokers concluded that long-term nontobacco smoking was associated with a reduction in $\mathrm{FEV}_{1}, \mathrm{Vmax}_{50}$, and $\mathrm{FEV}_{1} / \mathrm{FVC}^{9}{ }^{9}$ Of note, most of the studies that reported impaired lung function from marijuana smoking had enrolled active smokers who had at least 20 joints of exposure. ${ }^{9}$ 
The major limitations of these epidemiologic and clinical studies can be distilled to three large domains: small samples and short follow-up times; possible confounding by cigarette smoke, as most "hard-core" marijuana smokers in the past also smoked tobacco; and challenges in accurately measuring "exposure" of marijuana smoke. The importance of follow-up time cannot be understated. Lung cancer and chronic obstructive pulmonary disease (COPD), two major health concerns for marijuana smokers, take many decades to develop and usually do not manifest clinically until the fifth or sixth decades of life. Moreover, even if marijuana smoke has similar harmful effects on the airways as tobacco smoke, it is very likely that only a relatively small proportion (10\%-20\%) of active marijuana smokers would develop these clinical conditions. Thus, large samples of "susceptible" individuals (e.g., active smokers aged $>40 \mathrm{yr}$ ) would be required to show convincingly the independent effects of marijuana smoke on the risk of COPD and lung cancer in the population.

It is also important to develop new tools for accurately assessing exposure among marijuana smokers. Self-report alone is inadequate; it likely underestimates true marijuana smoking exposure owing to reporting and recall biases. Furthermore, as there is tremendous variation in cultural patterns of marijuana smoking, strength of marijuana strains and intensity of smoking between individuals, there is an urgent need to develop sensitive biomarkers to quantify accurately the extent of exposure among smokers. Ideally, these marijuana biomarkers should be independent of biomarkers for cigarette smoke (e.g., plasma cotinine), to enable clear separation of exposures. Moreover, it is important to distinguish acute effects of marijuana smoke on lung function from their chronic effects. The findings of experimental research have led to speculation that the failure to show consistent associations of marijuana smoking with impairment in lung function could be due to the ameliorating effects of acute marijuana exposure on airway smooth muscle, which leads to bronchorelaxation and improved lung function, and the possible immunosuppressive effect of THC, which results in less airway inflammation. ${ }^{7}$

Better research is needed to refine public health discourse and policies regarding marijuana smoking. It is essential that, with the legalization of marijuana, well-designed preclinical in vitro, animal and clinical human studies are conducted to address pressing gaps in knowledge, including quantification and composition of commercially available marijuana; development of biomarkers for detection of current and past use of marijuana; quantitative comparison of unit-standardized marijuana cigarette and tobacco cigarette; and data on alternative devices or methods for marijuana delivery and their effect on health. With further research, the mysteries and myths behind marijuana can be resolved.

\section{References}

1. Schluttenhofer C. Canada begins a great ganja experiment. Science 2018;361:460.

2. Cannabis legalization and regulation. Ottawa: Government of Canada, Department of Justice; (modified 2018 Aug. 9). Available: www.justice.gc.ca/eng/cj-jp/ cannabis (accessed 2018 Oct. 5).

3. The reports of the Surgeon General: the 1964 report on smoking and Health. Bethesda (MD): U.S. National Library of Medicine; 1964 Jan. 11. Available: https:// profiles.nlm.nih.gov/ps/retrieve/Narrative/NN/p-nid/60 (accessed 2018 Oct. 1).

4. Hall W. Minimizing the adverse public health effects of cannabis legalization. CMAJ 2018;190:E1031-2.

5. Derbyshire D. Smoking is 'good for your memory and concentration'. Daily Mail; 2008 Aug. 14. Available: www.dailymail.co.uk/health/article-1034701/ Smoking-good-memory-concentration.html (accessed 2018 Oct. 1).

6. Drug and Therapeutics Bulletin. Republished: Nicotine and health. BMJ 2014;349:2014.7.0264rep.

7. Moir D, Rickert WS, Levasseur G, et al. A comparison of mainstream and sidestream marijuana and tobacco cigarette smoke produced under two machine smoking conditions. Chem Res Toxicol 2008;21:494-502.

8. Maertens RM, White PA, Williams A, et al. A global toxicogenomic analysis investigating the mechanistic differences between tobacco and marijuana smoke condensates in vitro. Toxicology 2013;308:60-73.

9. Biehl JR, Burnham EL. Cannabis smoking in 2015: A concern for lung health? Chest 2015;148:596-606.

10. Ghasemiesfe M, Ravi D, Vali M, et al. Marijuana use, respiratory symptoms, and pulmonary function: a systematic review and meta-analysis. Ann Intern Med 2018;169:106-15.
Competing interests: Wan Tan reports personal fees from Teva and GlaxoSmithKline. Don Sin reports grants from AstraZeneca and Merck, personal fees from Sanofi-Aventis, Regeneron and Novartis, and grants and personal fees from Boehringer Ingelheim and AstraZeneca,

This article was solicited and has not been peer reviewed.

Affiliations: Centre for Heart Lung Innovation (Tan, Sin), University of British Columbia; St. Paul's Hospital (Tan, Sin), Vancouver, BC
Contributors: Both authors contributed to the conception and implementation of the commentary, the interpretation of data and the writing of the manuscript and its revision. Both authors approved the final version of the manuscript and agreed to be accountable for all aspects of the work.

Correspondence to: Wan Tan, wan.tan@hli.ubc.ca 